\title{
Database of the MANSCAPE project (Management tools for water bodies in agricultural landscapes)
}

Pieter Lemmens, Aaike De Wever, Henrietta Hampel, Tom De Bie, Dirk Ercken, Jeroen Van Wichelen, Luc Denys, Boudewijn Goddeeris, Syaghalirwa N.M. Mandiki, Leo Vanhecke, Katleen van der Gucht, Dirk Bauwens, Sara Denayer, Riet Durinck, Renaat Dasseville, Marie Lionard, Frank van De Meutter, Gerald Louette, Ann Hulsmans, Koen De Gelas, Isa Schön, Hilde Vrijders, Annelies Maes, Bertrand Losson, Saadia Lasri, Patrick Kestemont, Wim Vyverman, Pieter Vanormelingen, Luc Brendonck, Luc De Meester, Steven A.J. Declerck \& Koen Martens

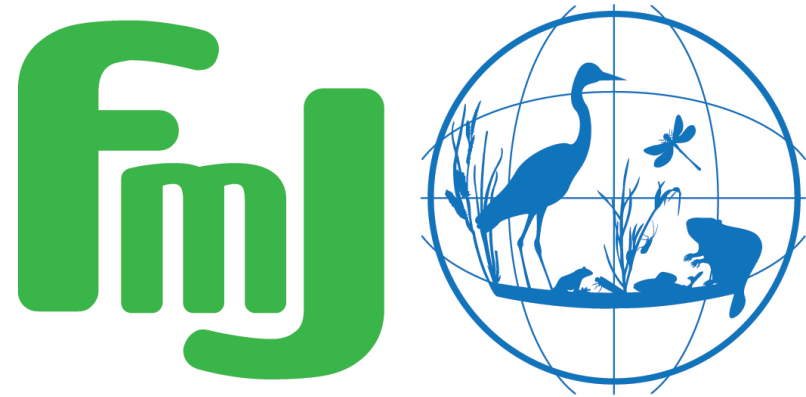

Freshwater Metadata Journal 



\section{Database of the MANSCAPE project (Management tools for water bodies in agricultural landscapes)}

Pieter Lemmens ${ }^{1}$, Aaike De Wever ${ }^{2}$, Henrietta Hampel ${ }^{2}$, Tom De Bie ${ }^{1}$, Dirk Ercken ${ }^{1}$, Jeroen Van Wichelen ${ }^{3}$, Luc Denys ${ }^{4}$, Boudewijn Goddeeris ${ }^{2}$, Syaghalirwa N.M. Mandiki ${ }^{5}$, Leo Vanhecke ${ }^{6}$, Katleen van der Gucht ${ }^{3}$, Dirk Bauwens ${ }^{4}$, Sara Denayer ${ }^{3}$, Riet Durinck ${ }^{3}$, Renaat Dasseville ${ }^{3}$, Marie Lionard ${ }^{3}$, Frank van De Meutter ${ }^{1}$, Gerald Louette ${ }^{1}$, Ann Hulsmans ${ }^{1}$, Koen De Gelas ${ }^{1}$, Isa Schön ${ }^{2}$, Hilde Vrijders ${ }^{2}$, Annelies Maes ${ }^{2}$, Bertrand Losson $^{7}$, Saadia Lasri ${ }^{7}$, Patrick Kestemont ${ }^{5}$, Wim Vyverman ${ }^{3}$, Pieter Vanormelingen ${ }^{3}$, Luc Brendonck ${ }^{1}$, Luc De Meester ${ }^{1}$, Steven A.J. Declerck ${ }^{1}$ \& Koen Martens ${ }^{2}$

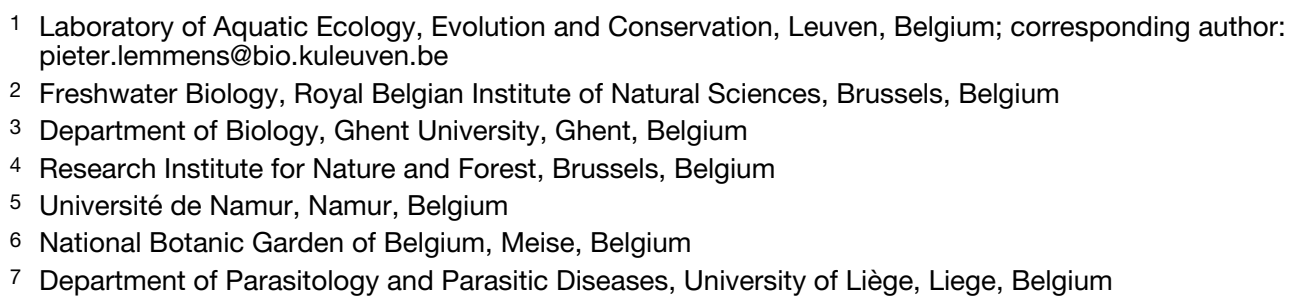

Please cite this paper as follows: Lemmens, P., De Wever, A., Hampel, H., De Bie, T., Ercken, D., Van Wichelen, J., Denys, L., Goddeeris, B., Mandiki, S.N.M, Vanhecke, L., van der Gucht, K., Bauwens, D., Denayer, S., Durinck, R., Dasseville, R., Lionard, M., van De Meutter, F., Louette, G., Hulsmans, A., De Gelas, K., Schön, I., Vrijders, H., Maes, A., Losson, B., Lasri, S., Kestemont, P., Vyverman, W., Vanormelingen, P., Brendonck, L., De Meester, L., Declerck, S.A.J. \& Martens, K., 2017. Database of the MANSCAPE project (Management tools for water bodies in agricultural landscapes). Freshwater Metadata Journal 26: 1-11. https://doi.org/10.15504/fmj.2017.26

Received: 2017-08-11 / Published: 2017-10-11

\section{Keywords}

farmland ponds, occurrences, environmental conditions, Belgium

\section{Short description of the dataset/summary}

The database of the MANSCAPE project (Integrated management tools for water bodies in agricultural landscapes) comprises species occurrence data of seven different organism groups (phytoplankton, diatoms, zooplankton, macro-invertebrates, macrophytes, amphibians and fish) and data on physical, chemical and morphometric variables of 126 small farmland ponds distributed over almost the entire Belgian territory. 


\section{General information}

dataset entry ID:

name of the dataset:

full name of the dataset:

dataset short name:

type of dataset:

data type:

science keywords according to GCMD:

\section{SF_2}

Integrated management tools for water bodies in agricultural landscapes MANSCAPE

species (taxonomic group) per site database including environmental information

point data/observation data topic:

ISO topic category according to ISO 19115 :

own science keywords:

Farming, Biota, Environment, Inland Waters

farmland ponds, occurrences, environmental conditions, Belgium

\section{Technical and administrative specifications}

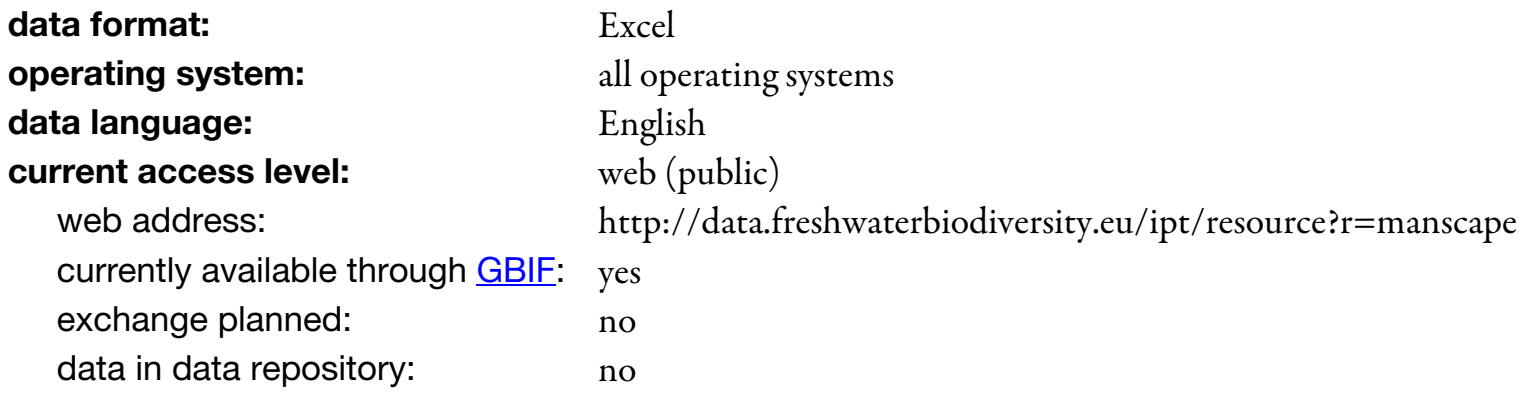

Excel

all operating systems

English

web (public)

http://data.freshwaterbiodiversity.eu/ipt/resource?r=manscape

yes

no

no

Do you plan to publish the data on the Freshwater Biodiversity Data Portal:

update level:

documentation:

type:

language:

\section{contact details:}

metadata contact person:

first, last name:

phone:

email:

institution:

address:

postal code, city:

country

technical contact person:

first, last name:

phone:

email:

scientific contact person:

first, last name:

phone:

email: already published through the Freshwater Biodiversity Data Portal

completed

scientific paper

English

Koen Martens

+32(0)26274315

koen.martens@naturalsciences.be

Royal Belgian Institute of Natural Sciences

Vautierstraat 29

1000 Brussels

Belgium

Koen Martens

+32(0)26274315

koen.martens@naturalsciences.be

Koen Martens

+32(0)26274315

koen.martens@naturalsciences.be 


\section{Intellectual property rights and citation}

dataset creator (data compiler):

contact name:

Pieter Lemmens

contact email:

contact institution:

pieter.lemmens@bio.kuleuven.be

KU Leuven

data contributors to/owners of this dataset:

number:

multiple

6

\section{data contributor/owner 1:}

contact name:

Koen Martens

contact email:

contact institute:

koen.martens@naturalsciences.be

KBIN

criteria for using this part of the dataset:

The dataset is publicly available (data portal, data archive) and can be used without restrictions, but dataset creator/data contributors must be informed prior to publication. Data must be acknowledged and cited correctly.

data contributor/owner 2:

contact name:

Wim Vijverman

contact email:

contact institute:

wim.vyverman@ugent.be

UGent

criteria for using this part of the dataset:

The dataset is publicly available (data portal, data archive) and can be used without restrictions, but dataset creator/data contributors must be informed prior to publication. Data must be acknowledged and cited correctly.

data contributor/owner 3:

contact name:

Patrick Kestemont

contact email:

patrick.kestemont@unamur.be

contact institute:

UNamur

criteria for using this part of the dataset:

The dataset is publicly available (data portal, data archive) and can be used without restrictions, but dataset creator/data contributors must be informed prior to publication. Data must be acknowledged and cited correctly.

data contributor/owner 4:

contact name:

Dirk Bauwens

contact email:

dirk.bauwens@inbo.be

contact institute:

INBO

criteria for using this part of the dataset:

The dataset is publicly available (data portal, data archive) and can be used without restrictions, but dataset creator/data contributors must be informed prior to publication. Data must be acknowledged and cited correctly.

data contributor/owner 5:

contact name:

contact email:

contact institute:

criteria for using this part of the dataset:

Leo Van Hecke

leo.vanhecke@skynet.be

National Botanical Garden

The dataset is publicly available (data portal, data archive) and can be used without restrictions, but dataset creator/data contributors must be informed prior to publication. Data must be acknowledged and cited correctly.

data contributor/owner 6:

contact name:

Luc De Meester 
contact email: luc.demeester@kuleuven.be

contact institute: KULeuven

criteria for using this part of the dataset:

The dataset is publicly available (data portal, data archive) and can be used without restrictions, but dataset creator/data contributors must be informed

citation of this dataset: prior to publication. Data must be acknowledged and cited correctly.

author(s):

Lemmens, P., De Wever, A., Hampel, H., De Bie, T., Ercken, D., Van Wichelen, J., Denys, L., Goddeeris, B., Mandiki, S.N.M., van Hecke, L., van der Gucht, K., Bauwens, D., Durinck, R., Dasseville, R., Lionard, M., van De Meutter, F., Louette, G., Hulsmans, A., De Gelas, K., Schön, I., Vrijders, H., Maes, A., Losson, B., Lasri, S., Kestemont, P., Vyverman, W., Vanormelingen, P., Brendonck, L., De Meester, L., Declerck, S.A.J. \& Martens, K.

title and journal (name, number, pages):

Management tools for waterbodies in agricultural landscapes (MANSCAPE)

year:

2017

doi:

https://doi.org/10.13148/6hacwh

citation of the metadata:

author(s):

Lemmens P., De Wever A., Hampel H., De Bie T., Ercken D., Van Wichelen J., Denys L., Goddeeris B., Mandiki S.N.M, Vanhecke L., van der Gucht K.,

Bauwens D., Denayer S., Durinck R., Dasseville R., Lionard M., van De Meutter F., Louette G., Hulsmans A., De Gelas K., Schön I., Vrijders H., Maes A., Losson B., Lasri S., Kestemont P., Vyverman W., Vanormelingen P., Brendonck L., De Meester L., Declerck S.A.J. \& Martens K.

title and journal (name, number, pages):

Database of the MANSCAPE project (Management tools for water bodies in agricultural landscapes). Freshwater Metadata Journal 26: 1-11

year: 2017

doi:

https://doi.org/10.15504/fmj.2017.26

\section{dataset related references:}

reference 1:

author(s):

De Bie T., De Meester L., Brendonck L., Martens K., Goddeeris B., Ercken D., Hampel H., Denys L., Vanhecke L., Van der Gucht K., Van Wichelen J., Vyverman W., Declerck S.L., Van der Gucht K., Van Wichelen J., Vyverman W. \& Declerck S.A.J.

title:

Body size and dispersal mode as key traits determining metacommunity structure of aquatic organisms. Ecology Letters, 15(7), 740-747.

year: 2012

doi: $\quad$ https://doi.org/doi: $10.1111 / j .1461-0248.2012 .01794 . x$

\section{General data specifications}

regional coverage of the dataset:

spatial extent of the dataset:

national

continents:

spatial extent (bounding coordinates):

southernmost latitude $\left[^{\circ}\right]$ :

Europe

northernmost latitude $\left[^{\circ}\right]$ :

51.42

westernmost longitude $\left[^{\circ}\right]$ :

49.68

2.84 
easternmost longitude $\left[^{\circ}\right]$ :

countries:
5.95

Europe: Belgium

world climatic regions according to Köppen:

Group C: temperate/mesothermal climates

freshwater ecoregions of the world (FEOW) according to WWF:

Europe: Central \& Western Europe

European ecoregions according to Illies (WFD):

ecosystem type:

covered timeframe:
Western Plains (ER13)

lakes/ponds

$2003-2004$

\section{Site specifications}

$\begin{array}{ll}\text { coordinate system/grid data: } & \begin{array}{l}\text { latitude/longitude, format: DD } \\ \text { projected, UTM }\end{array} \\ \text { datum (e.g. WGS84): } & \text { WGS84 } \\ \text { grid data available: } & \text { no } \\ \text { site coding: } & \\ \text { site coding available: } & \text { yes, alphanumerical } \\ \text { number of digits: } & 7 \\ \text { example: } & \text { AnHerEx } \\ \text { number of sites: } & 100-1000 \\ \text { exact number of sites: } & 126\end{array}$

\section{Climate and environmental data}

climate related data:

environmental data:

available parameters per site:

comments: no climate data available

no environmental data per catchment available

maximum depth

mean depth

substrate composition

land use at multiple perimeters around each pond

Data on land use in different perimeters around the pond are available in the main database (see also T. De Bie, L. De Meester, L. Brendonck, K. Martens, B. Goddeeris, D. Ercken, H. Hampel, L. Denys, L. Vanhecke, K. Van der Gucht, J. Van Wichelen, W. Vyverman \& S. A. J. Declerck (2012): Body size and dispersal mode as key traits determining metacommunity structure of aquatic organisms. Ecology Letters, 15(7), 740-747.)

total $\mathrm{P}$, nitrate, total $\mathrm{N}$, sulphate, hardness, alkalinity, oxygen content, water temperature, $\mathrm{pH}$, conductivity, chlorophyll, Secci disc depth, suspended solids availability of physico-chemical data, if there is more than one sample per site:

stressors influencing the sites:

reference sites available: per sample

no stressor data available

no 


\section{Biological data}

biological data origin:

organism group addressed: from sampling, MANSCAPE

amphibians, fish, macro-invertebrates (Mollusca, Ephemeroptera, Odonata, Plecoptera, Coleoptera, Trichoptera, Chironomidae), zooplankton (Cladocera), phytoplankton, (benthic) diatoms, macrophytes

\section{Sample specifications/sample resolution}

\section{amphibians: \\ sample information: \\ covered timeframe: \\ historical data: \\ season: \\ time series data: \\ taxonomic resolution: \\ level: \\ taxonomic coding: \\ taxalist according to: \\ reference(s): \\ sample specifications: \\ type: \\ replicate samples: \\ comments:}

temporal resolution/frequency of sampling:

no

summer

per year

no

species

no
$2003-2003$

Arnold E.N. \& Ovenden D.W. (2002)

Arnold E.N. \& Ovenden D.W. (2002). Reptiles and amphibians of Europe.

Princeton University Press, Princeton, NJ.

presence/absence

For details see appendix of T. De Bie, L. De Meester, L. Brendonck, K. Martens, B. Goddeeris, D. Ercken, H. Hampel, L. Denys, L. Vanhecke, K. Van der Gucht, J. Van Wichelen, W. Vyverman \& S. A. J. Declerck (2012) Body size and dispersal mode as key traits determining metacommunity structure of aquatic organisms. Ecology Letters, 15(7), 740-747.

fish:

sample information:

covered timeframe:

$2003-2003$

historical data:

no

palaeo data:

no

season:

summer

temporal resolution/frequency of sampling:

time series data:

per year

no

\section{taxonomic resolution:}

level:

species

\section{taxonomic coding:}

taxalist according to:

reference(s):

Keith P. \& Allardi J. (2001)

Keith P. \& Allardi J. (2001). Atlas des poissons d'eau douce de France. 
sample specifications:

type:

comments:

\section{macro-invertebrates:} sample information:

covered timeframe:

historical data:

palaeo data:

season:

no

no

summer

temporal resolution/frequency of sampling:

time series data:

taxonomic resolution:

level:

comments:

\section{taxonomic coding:}

taxalist according to:

reference(s):

\section{sample specifications:}

type:

replicate samples:

comments:

per year

no

species

no
Publications Scientifiques du M.N.H.N., Paris.

quantitative (abundance data)

For details see appendix of T. De Bie, L. De Meester, L. Brendonck, K. Martens,

B. Goddeeris, D. Ercken, H. Hampel, L. Denys, L. Vanhecke, K. Van der Gucht, J. Van Wichelen, W. Vyverman \& S. A. J. Declerck (2012) Body size and dispersal mode as key traits determining metacommunity structure of aquatic organisms. Ecology Letters, 15(7), 740-747.

Chironomidae, Ephemeroptera, Trichoptera, Heteroptera, Mollusca and Coleoptera were identified to species level.

see full reference list below

Devriese R., Warmoes T. \& Vercoutere B. (1997). Land- en zoetwatermollusken van de Benelux. Jeugdbond voor Natuur en Milieu, Gent.

De Pauw N. \& Vannevel R. (1990). Macro-invertebraten en waterkwaliteit. Stichting Leefmilieu vzw., Antwerpen.

Drost M.B.P., Cuppen H.P.J.J., Nieukerken E.J. \& Schreijer M. (1992). De waterkevers van Nederland. Uitgeverij K.N.N.V., Utrecht, The Netherlands.

Savage A.A. (1989). Adults of the British aquatic Hemiptera Heteroptera: a key with ecological notes. Freshwater Biological Association, Ambleside (UK).

Wiederholm T. (1983). Chironomidae of the holarctic region: keys and diagnoses, Part 1 - larvae. Entomologica Scaninavica Supllement 19.

Moller Pillot H.K.M. (1984a). De larven der Nederlandse Chironomidae (Diptera) (Inleiding,Tanypodinae en Chironomini). Nederlandse Faunistische Mededelingen, 1A, 1-227.

Moller Pillot H.K.M. (1984b). De larven der Nederlandse Chironomidae (Diptera) (Orthocladiinae sensu lato). Nederlandse Faunistische Mededelingen, IB, 1-175.

quantitative (abundance data)

For details see appendix of T. De Bie, L. De Meester, L. Brendonck, K. Martens, B. Goddeeris, D. Ercken, H. Hampel, L. Denys, L. Vanhecke, K. Van der Gucht, J. Van Wichelen, W. Vyverman \& S. A. J. Declerck (2012) Body size and 
dispersal mode as key traits determining metacommunity structure of aquatic organisms. Ecology Letters, 15(7), 740-747.

\section{zooplankton:}

sample information:

covered timeframe:

historical data:

palaeo data:

season:

temporal resolution/frequency of sampling:

time series data:

taxonomic resolution:

level:

comments:

\section{taxonomic coding:}

taxalist according to:

reference(s):

no

no

summer

per year

no

species

\section{sample specifications: \\ type: \\ replicate samples: \\ comments:}

$2003-2003$

Cladocera and Rotifera were counted and identified to species and genus level, respectively.

Nootenboom-Ram E. (1981); Flössner D. (2000); Alonso M. (1996)

Nootenboom-Ram E. (1981). Verspreiding en Ecologie van de Branchiopoda in Nederland.In: Arnhem, p. 95.

Alonso M. (1996). Crustacea Branchiopoda. Fauna Iberica 7. Museo Nacional de Ciencias Naturales (CSIC) Madrid.

Flössner D. (2000). Die Haplopoda und Cladocera (ohne Bosminidae)

Mitteleuropas. Backhuys Publishers, Leiden.

quantitative (abundance data)

no

For details see appendix of T. De Bie, L. De Meester, L. Brendonck, K. Martens, B. Goddeeris, D. Ercken, H. Hampel, L. Denys, L. Vanhecke, K. Van der Gucht, J. Van Wichelen, W. Vyverman \& S. A. J. Declerck (2012) Body size and dispersal mode as key traits determining metacommunity structure of aquatic organisms. Ecology Letters, 15(7), 740-747.

$2003-2003$

no

summer

temporal resolution/frequency of sampling:

taxonomic resolution:

per year

genus

taxonomic coding:

taxalist according to:

reference(s):

sample specifications:

type:
John D.M., Whitton B.A. \& Brook A.J. (2002); Tikkanen T. \& Willén T. (1992)

John D.M., Whitton B.A. \& Brook A.J. (2002). The freshwater algal flora of the British isles. Cambridge University Press, Cambridge.

Tikkanen T. \& Willén T. (1992). Växtplantonflora. Författarna Natur, Solna 
replicate samples:

comments: no

For details see appendix of T. De Bie, L. De Meester, L. Brendonck, K. Martens, B. Goddeeris, D. Ercken, H. Hampel, L. Denys, L. Vanhecke, K. Van der Gucht, J. Van Wichelen, W. Vyverman \& S. A. J. Declerck (2012) Body size and dispersal mode as key traits determining metacommunity structure of aquatic organisms. Ecology Letters, 15(7), 740-747.

\section{(benthic) diatoms: \\ sample information:}

covered timeframe:

$2003-2004$

historical data:

no

palaeo data:

no

season:

summer

temporal resolution/frequency of sampling:

time series data:

per year

no

\section{taxonomic resolution:}

level:

taxonomic coding:

taxalist according to:

reference(s): species

see full reference list below

Krammer K. \& Lange-Bertalot H. (1986). Süßwasserflora von Mitteleuropa 2: Bacillariophyceae. 1. Teil: Naviculaceae. Gustav Fischer Verlag, Stuttgart.

Krammer K. (1997a). Die cymbelloiden Diatomeen. Eine Monographie der weltweit bekannten Taxa. Teil 1. Algemeines und Encyonema Part. Bibliotheca Diatomologica 36, 1-382.

Krammer K. (1997b). Die cymbelloiden Diatomeen. Eine Monographie der weltweit bekannten Taxa. Teil 2. Encyonema part., Encyonopsis and Cymbellopsis. Bibliotheca Diatomologica 37, 1-469.

Krammer K. (2000). Diatoms of Europe. Vol. 1. The genus Pinnularia. A.R.G. Gantner Verlag K.G., Ruggell.

Krammer K. (2002). Diatoms of Europe. Vol. 3. Cymbella. A.R.G. Ganter Verlag K.G., Ruggell.

Krammer K. (2003). Diatoms of Europe. Vol. 4. Cymbopleura, Delicata, Navicymbula, Gomphocymbellopsis, Afrocymbella. A.R.G. Ganter Verlag K.G., Ruggell.

Krammer K. \& Lange-Bertalot H. (2004). Süßwasserflora von Mitteleuropa 2: Bacillariophyceae. 3. Teil: Centrales, Fragilariaceae, Eunotiaceae. Elsevier GmbH, Spektrum Akademischer Verlag, Heidelberg.

Krammer K. \& Lange-Bertalot H. (2007). Süßwasserflora von Mitteleuropa 2: Bacillariophyceae. 2. Teil: Bacillariaceae, Epithemiaceae, Surirellaceae. Ergänzter Nachdruck der 1. Auflage. Elsevier GmbH, Spektrum Akademischer Verlag, Heidelberg.

Lange-Bertalot H. (2001). Diatoms of Europe. Vol. 2. Navicula sensu stricto, 10 


\section{sample specifications:}

type:

replicate samples:

comments:

genera separated from Navicula sensu lato, Frustulia. Gartner Verlag, Ruggel.

Lange-Bertalot H. \& Moser G. (1994). Brachysira: Monographie der Gattung. Bibliotheca Diatomologica 29, 1-212.

Lange-Bertalot H. \& Metzeltin D. (1996). Oligotrophie-Indikatoren. 800 Taxa repräsentativ für drei diverse Seen-Typen, kalkreich - oligodystroph - schwach gepuffertes Weichwasser. Iconographia Diatomologica 2: 1-390. 9

Lange-Bertalot H., Cavacini P., Tagliaventi N. \& Alfinito S. (2003). Diatoms of Sardinia. Rare and 76 new species in rock pools and other ephemeral waters. Iconographia Diatomologica 12, 1-438.

Werum M. \& Lange-Bertalot H. (2004). Diatoms in springs from Central Europe and elsewhere under the influence of hydrogeology and anthropogenic impacts. Iconographia Diatomologica 13, 3-417.

quantitative (abundance data)

no

For details see appendix of T. De Bie, L. De Meester, L. Brendonck, K. Martens, B. Goddeeris, D. Ercken, H. Hampel, L. Denys, L. Vanhecke, K. Van der Gucht, J. Van Wichelen, W. Vyverman \& S. A. J. Declerck (2012) Body size and dispersal mode as key traits determining metacommunity structure of aquatic organisms. Ecology Letters, 15(7), 740-747.

macrophytes:

sample information:

covered timeframe:

$2003-2003$

historical data:

no

palaeo data:

no

season:

summer

temporal resolution/frequency of sampling:

taxonomic resolution:

per year

level:

taxonomic coding:

taxalist according to:

reference(s):

species

see full reference list

Haslam S.M., Sinker C.A. \& Wolseley P.A. (1975). British water plants. Field Studies 4, 243-351.

Casper S.J. \& Kraus H.D. (1980). Süssenwasserflora von Mitteleuropa, Band 23. Pteridophyta und Anthophyta, 1. Teil: Lycopodiaceae bis Orchidaceae Fischer Verlag, Jena.

Jermy A.C., Chater A.O. \& David R.W. (1982). Sedges of the British Isles 2 edn. Botanical Society of the British Isles, London.

\section{sample specifications:}

type:

comments:

semi-quantitative

For details see appendix of T. De Bie, L. De Meester, L. Brendonck, K. Martens, B. Goddeeris, D. Ercken, H. Hampel, L. Denys, L. Vanhecke, K. Van der Gucht, J. Van Wichelen, W. Vyverman \& S. A. J. Declerck (2012) Body size and dispersal mode as key traits determining metacommunity structure of aquatic 
organisms. Ecology Letters, 15(7), 740-747.

\section{Other specifications}

GIS layers, shape files related to the dataset:

$\begin{array}{ll} & \text { no data available } \\ \text { availability of photos: } & \text { no } \\ \text { availability of maps: } & \text { no } \\ \text { quality control procedures: } & \end{array}$

Were any quality control procedures applied to your dataset?

yes

quality control protocols and comments:

Species names were checked using the GBIF species list. In addition, the quality of data has been checked with the phwhip validator.

\section{Acknowledgements}

The authors acknowledge the Belgian Science Policy (Belspo) for funding the original MANSCAPE project (Integrated management tools for water bodies in agricultural landscapes" - 2003-2006), as well as for funding the SAFRED project (Saving freshwater biodiversity research data - 2015-2018) that allowed processing and publishing the Manscape data, amongst others.

\section{References}

De Bie T., De Meester L., Brendonck L., Martens K., Goddeeris B., Ercken D., Hampel H., Denys L., Vanhecke L., Van der Gucht K., Van Wichelen J., Vyverman W., Declerck S.L., Van der Gucht K., Van Wichelen J., Vyverman W. \& Declerck S.A.J., 2012. Body size and dispersal mode as key traits determining metacommunity structure of aquatic organisms. Ecology Letters 15(7), 740-747. https://doi.org/10.1111/j.1461-0248.2012.01794.x 